\title{
Avaliação de Unidade de Medição Inercial Utilizando Técnicas de Reconstrução de Trajetória de Pedestres
}

\author{
Rodolfo Nícolas Rocha e Silva \\ Departamento de Estatística e Informática \\ Universidade Federal Rural de Pernambuco \\ Recife, Brasil \\ pajaraca.n@gmail.com
}

\author{
Glauco Estácio Gonçalves \\ Instituto de Tecnologia \\ Universidade Federal do Pará \\ Belém, Brasil \\ glaucogoncalves@ufpa.br
}

\author{
Victor Wanderley Costa de Medeiros \\ Departamento de Estatística e Informática \\ Universidade Federal Rural de Pernambuco \\ Recife, Brasil \\ victor.wanderley@ufrpe.br
}

\begin{abstract}
Diversos trabalhos propuseram métodos para reconstruir trajetória e estimar a posição de pedestres baseando-se em unidades de medição inercial. Montando os sensores no pé é possível utilizar um filtro de Kalman estendido e com a técnica ZUPT lidar com os erros oriundos das medições dos sensores. Este trabalho busca analisar a influência das configurações de uma unidade de medição inercial de baixo custo nos resultados finais desses sistemas. As configurações dos sensores, relacionadas ao fundo de escala e sensibilidade de medição, foram estudadas comparando estatisticamente os erros de diferentes métodos de reconstrução de trajetória. Os resultados apontam o fundo de escala do giroscópio como o parâmetro mais influente do sistema, já a combinação configuração e algoritmo com melhor desempenho apresentam uma média dos erros de posicionamento de $0.98 \%$ da distância total percorrida.
\end{abstract}

Index Terms-pedestrian dead reckoning, inertial measurement unit, ZUPT, extended Kalman filter, Madgwick, sistema de navegação inercial

\section{InTRODUÇÃO}

Estimar a posição de uma pessoa ou o caminho percorrido por ela é um problema de interesse em várias áreas. Um bombeiro em um prédio em chamas, um consumidor em um centro de compras, um viajante numa estrada, um participante de uma corrida são alguns dos exemplos de situações onde estas estimativas permitem a construção de soluções inovadoras. A precisão de um GPS é suficiente em vários casos, porém existem situações em que esse dispositivo não será preciso o suficiente, sofrerá interferências de outros equipamentos, ou não haverá sinal disponível como em prédios, túneis ou florestas. A posição de um pedestre pode ser estimada a partir de sensores inerciais, utilizando técnicas de navegação inercial e dead-reckoning, não sendo necessários sinais externos ou uma infraestrutura pré-estabelecida. Essas estratégias são geralmente chamadas de pedestrian dead-reckoning (PDR), ou pedestrian inertial navigation system (PINS), nelas os sinais dos acelerômetros são integrados duas vezes para estimar a posição e os sinais dos giroscópios são integrados para estimar a orientação. Sensores inerciais estão sujeitos a erros, que se acumulam e aumentam rapidamente devido as sucessivas integrações e as próprias características dos sensores. Sensores de baixo custo são ainda mais suscetíveis a esses erros, tornando necessárias estratégias de controle e o entendimento das influências desses erros nos resultados dos sistemas.

Um PDR com sensores montados no pé foi proposto em [1], onde a mecânica da caminhada humana foi utilizada na estratégia de controle dos erros do sistema. O sistema infere zero velocidade a cada vez que o pé está fixo no chão durante a caminhada, e alimenta um filtro de Kalman estendido (EKF) que estima os erros a cada passo para corrigir posição e velocidade, impedindo o crescimento descontrolado dos erros que inviabilizariam o sistema. A estratégia de zero velocidade ficou conhecida como zero velocity update (ZUPT). Esse EKF, trabalha no espaço dos erros, e após cada atualização realimenta o sistema de navegação inercial (SNI) com a estimativa dos erros computados. Baseado em [1], [2] apresentou um PDR chamado INS-EKF-ZUPT (IEZ) de 15 estados, onde foram integrados diversos métodos para reduzir erros na orientação, principalmente em yaw, como o zero angular rate update (ZARU) (proposto por [3]), heuristic heading reduction (HDR) e magnetômetros. Neste mesmo trabalho são apresentados detalhes sobre a detecção de zero velocidade do pé baseando-se em 3 condições. A múltipla condição para determinação da estacionariedade do pé também pode ser vista em [4] e [5]. Em [6] um PDR com EKF de 9 estados e ZUPT é proposto, num sistema similar a [7] que, entretanto, utiliza quatérnios para representação da orientação do sistema. Outros trabalhos como [4] e [8] também utilizaram o quatérnio para representar a orientação. Em [9] a abordagem tradicional de PDRs, com integração das velocidades angulares para estimativa das orientações, foi comparada com estimativas oriundas de filtros complementares como Madgwick [10] e Mahony [11].

Este trabalho apresenta uma análise das influências e impactos das configurações da unidade de medição inercial (IMU) de baixo custo MPU-6050 nas reconstruções de trajetória utilizando técnicas de PDR, com o objetivo de identificar o desempenho das configurações e compará-las. São avaliadas as configurações combinadas com 4 algoritmos: IEZ de 9 estados, IEZ+ZARU de 15 estados, IEZ+Madgwick 
de 9 estados e IEZ+ZARU+Madgwick de 15 estados. Para as análises foram realizadas 30 caminhadas em um circuito fechado para cada configuração da IMU. Os algoritmos processaram os dados, reconstruindo as trajetórias e extraindo os erros de posicionamento e de distância acumulada, que foram estudados e comparados estatisticamente.

\section{FUnDAMENTAÇÃO TEÓRICA}

\section{A. Sistema de navegação inercial}

Com uma IMU fixada a um corpo é possível estimar sua posição $(p)$, velocidade $(v)$ e orientação $(\theta)$ aplicando um algoritmo de navegação inercial strapdown (sistemas onde a IMU é fixada rigidamente ao corpo, as vezes chamado de amarrado). Velocidade e posição são obtidas após duas integrações sucessivas dos valores fornecidos pelos acelerômetros $\left(f^{b}\right)$, e a orientação é obtida a partir da integração dos valores fornecidos pelos giroscópios $\left(\omega^{b}\right)$.

A mecanização do sistema é dada por (1), (2) e (3), que são as equações de navegação que relacionam posição, velocidade e orientação.

$$
\begin{gathered}
\dot{p}^{n}=v^{n} \\
\dot{v}^{n}=C_{b}{ }^{n} f^{b}+g^{n} \\
\dot{C}_{b}{ }^{n}=C_{b}{ }^{n} \Omega
\end{gathered}
$$

Sendo que $b$ e $n$ representam o referencial do corpo (body-frame ou sensor-frame) e o referencial de navegação (navigation-frame ou global-frame) respectivamente, $C_{b}{ }^{n}$ é uma matriz $3 \times 3$ que define a orientação do referencial $b$ com relação ao referencial de navegação $n, f^{b}$ é o vetor que contêm a força específica no referencial do corpo, $\omega^{b}$ é o vetor que contêm a velocidade angular no referencial do corpo, $g^{n}$ é o vetor gravidade no referencial de navegação, e, $\Omega$ representa a matriz antissimétrica das velocidades angulares, dada por (4).

$$
\Omega=\left[\begin{array}{ccc}
0 & -\omega_{z}^{b} & \omega_{y}^{b} \\
\omega_{z}^{b} & 0 & -\omega_{x}^{b} \\
-\omega_{y}^{b} & \omega_{x}^{b} & 0
\end{array}\right]
$$

A discretização de (1), (2) e (3), para intervalos de tempo $\Delta_{t}$ entre as amostras $k$ e $k+1$, é dada por (5), (6), (7) e (8).

$$
\begin{gathered}
C_{b k+1}^{n}=C_{b k}^{n}\left(\frac{2 I_{3 \times 3}+\delta \Omega_{k+1} \Delta t}{2 I_{3 \times 3}-\delta \Omega_{k+1} \Delta t}\right) \\
f_{k+1}^{n}=C_{b k+1}^{n} f_{k+1}^{b}+g^{n} \\
v_{k+1}^{n}=v_{k}^{n}+f_{k+1}^{n} \Delta t \\
p_{k+1}^{n}=p_{k}^{n}+v_{k+1}^{n} \Delta t
\end{gathered}
$$

\section{B. Filtro de Kalman Estendido (EKF)}

Em um IEZ de 9 estados, Fig. 1, baseado em [6], o vetor que representa os erros do sistema é dado por (9). Sendo $\delta \phi$ os erros de orientação, $\delta p$ os erros de posição no referencial de navegação e $\delta v$ os erros de velocidade no referencial de navegação, cada um sendo um vetor com 3 elementos.

$$
\delta x=\left[\begin{array}{lll}
\delta \phi & \delta p & \delta v
\end{array}\right]
$$

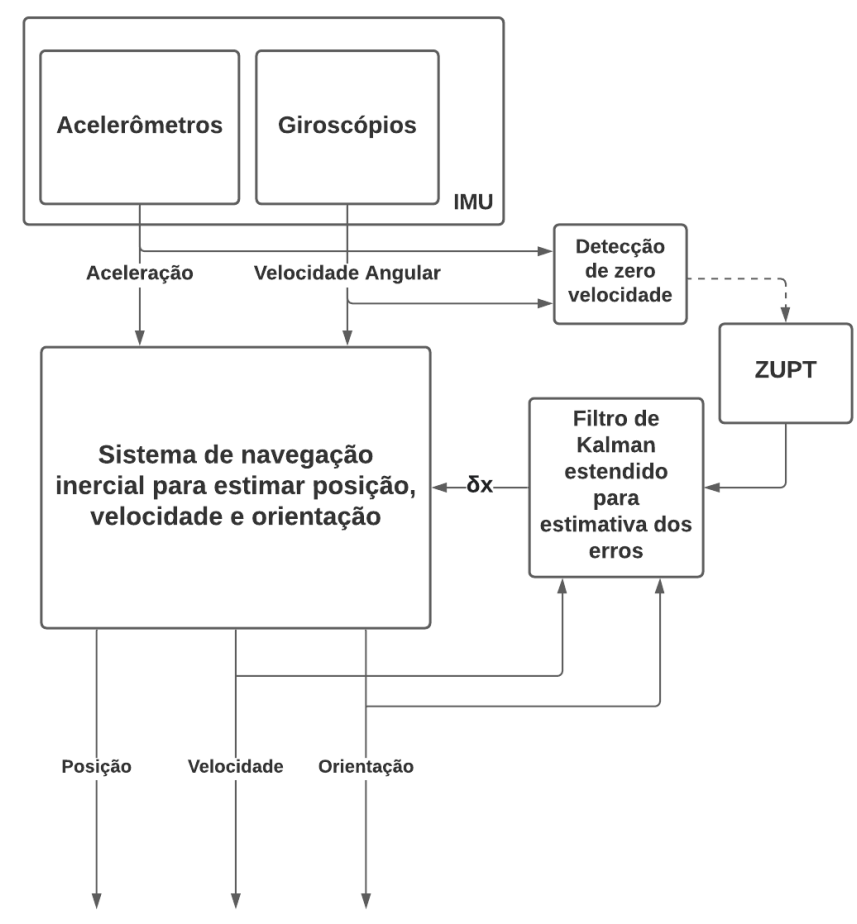

Fig. 1. IEZ de 9 estados para sensores montados no pé.

Como o modelo não é linear, (10) apresenta o modelo de transição linearizado. Onde $x_{k+1}$ é a estimativa do estado, $x_{k}$ é o estado do sistema no intervalo $k, \omega_{k}$ é o ruído de processo (de covariância $Q_{k+1}=E\left(\omega_{k+1} \omega_{k+1}^{T}\right)$ ) e $F_{k+1}$ é a matriz de transição de estados dada por (11).

$$
\delta x_{k+1}=F_{k+1} \delta x_{k}+\omega_{k}
$$

$$
F_{k+1}=\left[\begin{array}{ccc}
I_{3 x 3} & 0_{3 x 3} & 0_{3 x 3} \\
0_{3 x 3} & I_{3 x 3} & I_{3 x 3} \Delta t \\
-S_{k+1} \Delta t & 0_{3 x 3} & I_{3 x 3}
\end{array}\right]
$$

Em $F_{k+1}$ o termo $S_{k+1}$ é uma matriz antissimétrica (12) construída a partir $f_{k+1}^{n}$, que relaciona a estimativa de erros de orientação a partir das forças específicas com a estimativa de erros velocidade.

$$
S_{k+1}=\left[\begin{array}{ccc}
0 & -f_{z}^{n} & f_{y}^{n} \\
f_{z}^{n} & 0 & -f_{x}^{n} \\
-f_{y}^{n} & f_{x}^{n} & 0
\end{array}\right]
$$

$\mathrm{O}$ modelo de medição $z_{k+1}$ aplicado quando a condição de ZUPT é verdadeira é dado por (13). Sendo $H$ a matriz 
de medição dada por (14), e $n_{k+1}$ é o ruido de medição (de covariância $R_{k+1}=E\left(n_{k+1} n_{k+1}^{T}\right)$ ).

$$
\begin{aligned}
& \delta z_{k+1}=H \delta x_{k+1}+n_{k+1} \\
& H=\left[\begin{array}{lll}
0_{3 \times 3} & 0_{3 \times 3} & I_{3 \times 3}
\end{array}\right]
\end{aligned}
$$

A estimativa dos erros é computada para o estado $k+1$, por (15), quando a medição para esse intervalo estiver disponível. $K$ é o ganho de Kalman, calculado por (16) e $P_{k}$ é a estimativa da matriz de covariância do erro, calculada por (17).

$$
\begin{gathered}
\delta x_{k+1}=\delta x_{k}+K_{k}\left[m_{k}-H \delta x_{k}\right] \\
K_{k+1}=P_{k} H^{T}\left(H P_{k} H^{T}-R_{k+1}\right)^{-1} \\
P_{k}=F_{k} P_{k-1} F_{k}^{T}-Q_{k}
\end{gathered}
$$

Um IEZ de 15 estados, como em [2] segue os mesmos princípios apresentados para o de 9 estados, com o vetor do sistema dado por (18) e matriz de transição de estados dada por (19). Sendo $\delta \phi, \delta p$ e $\delta v$ já descritos e $\delta \omega^{b}, \delta a^{b}$ erros relacionados ao viés de medição (drift) dos giroscópios e acelerômetros, respectivamente. Em geral, estimar o drift dos sensores é incluído no vetor de estados dos sistemas com o objetivo de diminuir ainda mais a propagação dos erros inerentes aos sensores.

$$
\begin{gathered}
\delta x=\left[\begin{array}{lllll}
\delta \phi & \delta p & \delta v & \delta \omega^{b} & \delta a^{b}
\end{array}\right] \\
F_{k+1}=\left[\begin{array}{ccccc}
I_{3 x 3} & C_{b k}^{n} \Delta t & 0_{3 x 3} & 0_{3 x 3} & 0_{3 x 3} \\
0_{3 x 3} & I_{3 x 3} & 0_{3 x 3} & 0_{3 x 3} & 0_{3 x 3} \\
0_{3 x 3} & 0_{3 x 3} & I_{3 x 3} & I_{3 x 3} \Delta t & 0_{3 x 3} \\
-S_{k+1} \Delta t & 0_{3 x 3} & 0_{3 x 3} & I_{3 x 3} & C_{b k}^{n} \Delta t \\
0_{3 x 3} & 0_{3 x 3} & 0_{3 x 3} & 0_{3 x 3} & I_{3 x 3}
\end{array}\right]
\end{gathered}
$$

\section{Representação e estimativa da orientação}

As representações de orientação mais comuns para indicar transformações vetoriais dos referenciais $b$ para o $n$, são os ângulos de Euler, quatérnios e cossenos diretores. Em (20), $x^{i}$ é um vetor no referencial $i$ e $x^{b}$ um vetor no referencial $b$, sendo $C_{b}{ }^{i}$ a matriz de transformação do referencial $b$ para o $i$.

$$
x^{i}=C_{b}{ }^{i} x^{b}
$$

Uma propriedade importante das matrizes de transformação para a navegação inercial é vista em (21). Onde a transformação inversa de um referencial para outro é dada pela transposta dessa matriz.

$$
C_{b}{ }^{i}=C_{i}{ }^{b^{T}}
$$

1) Ângulos de Euler: A rotação de um corpo rígido pode ser descrita pelos 3 ângulos de Euler: roll $(\phi)$, pitch $(\theta)$ e yaw $(\psi)$ que representam rotações sucessivas ao longo de 3 eixos ortogonais, sendo $\psi$ a rotação sobre o eixo $z, \theta$ sobre $y$ e $\phi$ sobre $x$. Assim, (22) define a transformação $C_{b}{ }^{n}$.

$$
C_{b}{ }^{n}=\left[\begin{array}{cc}
\cos \theta \cos \psi & \operatorname{sen} \theta \operatorname{sen} \phi \cos \psi-\cos \phi \operatorname{sen} \psi \\
\cos \theta \operatorname{sen} \psi & \operatorname{sen} \theta \operatorname{sen} \phi \operatorname{sen} \psi+\cos \phi \cos \psi \\
-\sin \theta & \cos \theta \operatorname{sen} \phi \\
\operatorname{sen} \theta \cos \phi \cos \psi+\operatorname{sen} \phi \operatorname{sen} \psi \\
\operatorname{sen} \theta \cos \phi \operatorname{sen} \psi-\operatorname{sen} \phi \cos \psi \\
\cos \theta \cos \phi
\end{array}\right]
$$

2) Quatérnios e filtro de Madgwick: A rotação de um corpo rígido ou um referencial de coordenadas tridimensional podem ser representados por um número complexo de quatro dimensões, uma real $\left(q_{0}\right)$ e três complexas $\left(q_{1}, q_{2}\right.$ e $\left.q_{3}\right)$, chamado quatérnio (23) [10].

$$
q=\left[\begin{array}{llll}
q_{0} & q_{1} & q_{2} & q_{3}
\end{array}\right]
$$

A partir de um quatérnio é possível realizar a rotação de um vetor tridimensional $v$ de duas formas. A primeira é considerando o vetor um quatérnio de parte real nula e realizando as operações de (24), onde $\otimes$ representa o produto entre dois quatérnios $\mathrm{e} *$ representa o conjugado do quatérnio.

$$
v^{n}=q \otimes v^{b} \otimes q^{*}
$$

A segunda forma é convertendo o quatérnio em uma matriz de transformação, como mostra (25) e depois seguindo da mesma forma que (20).

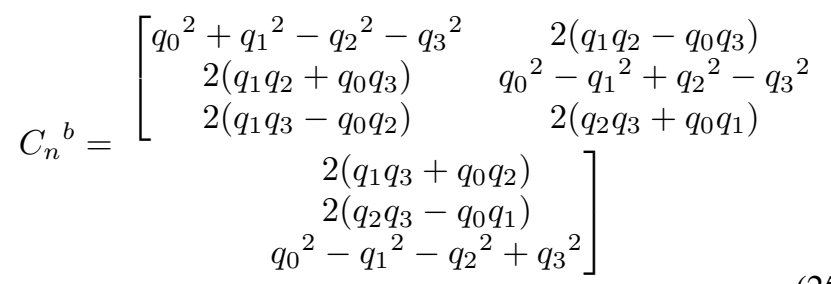

Em [9] foi apresentado um algoritmo capaz de fornecer a estimativa de orientação baseado nas medições de IMUs e MARGs (magnetic, angular rate and gravity) que ficou comumente conhecido como filtro de Madgwick.

No caso de IMUs a orientação inicial é calculada pela integração do quatérnio obtido das medições dos giroscópios, que depois é corrigida a partir da comparação com a medição do vetor gravidade pelos acelerômetros, onde se obtêm a estimativa final do quatérnio que representa a orientação, computado por (26). Em [9] e [12] esse processo é apresentado de forma simplificada.

$$
q_{t+1}=q_{t}+0.5 q_{t} \otimes \omega_{t} \Delta t-\beta \frac{\nabla f}{\|\nabla f\|} \Delta t
$$

Onde o termo $0.5 q_{t} \otimes \omega_{t} \Delta t$ é referente a atualização da orientação baseada na integração das velocidades angulares e 
$\frac{\nabla f}{\|\nabla f\|} \Delta t$ é o termo relacionado a correção com base nos dados dos acelerômetros e $\beta$ o peso atribuído a ele.

D. Detector de zero velocidade e métodos de redução de erro

É possível assumir a zero velocidade (ZV) do pé se valendo apenas dos dados obtidos pelos sensores inerciais. Em [4] foi utilizado um detector de $\mathrm{ZV}$ baseado unicamente na comparação do módulo de $\omega^{b}$ com um valor de referência. Apesar deste método obter resultados satisfatórios para vários casos, ainda sofre tanto com falhas na detecção quanto com falsas detecções. Para reduzir os problemas na detecção de ZV [2], [4], [5] e [8] utilizaram uma abordagem de múltiplas condições, que detecta a ZV apenas quando todas são satisfeitas simultaneamente.

Este trabalho utilizou duas condições (27) e (28) que devem ser satisfeitas simultaneamente para a detecção de ZV. O resultado das condições é depois filtrado utilizando uma janela de 10 medições.

$$
\begin{gathered}
C 1= \begin{cases}\left\|\omega^{b}\right\|<0.6 \mathrm{rad} / \mathrm{s} & 1 \\
\text { senão } & 0\end{cases} \\
C 2= \begin{cases}9 \mathrm{~m} / \mathrm{s}^{2}<\left\|f^{b}\right\|<11 \mathrm{~m} / \mathrm{s}^{2}, & 1 \\
\text { senão, } & 0\end{cases}
\end{gathered}
$$

Como já dito antes, é possível inferir que a velocidade do pé é zero quando, durante a caminhada, ele está em contato direto com o solo. Isso torna possível estimar o erro correspondente a velocidade naquele instante, que é a diferença entre a velocidade estimada e a pseudo-medição de ZV. Assim, é possível alimentar o EKF com esse erro e atualizar os estados do sistema, na técnica conhecida como ZUPT.

De forma similar a descrita anteriormente é possível inferir que a velocidade angular nesse mesmo momento também é zero e compará-la com os valores do sistema para alimentar o EKF [13] [14]. Essa técnica é conhecida como zero angular rate update (ZARU) e, quando utilizada em conjunto com o ZUPT, o sistema tem a matriz de medição dada por (29).

$$
H=\left[\begin{array}{ccccc}
0_{3 \times 3} & 0_{3 \times 3} & I_{3 \times 3} & 0_{3 \times 3} & 0_{3 \times 3} \\
0_{3 \times 3} & 0_{3 \times 3} & 0_{3 \times 3} & I_{3 \times 3} & 0_{3 \times 3}
\end{array}\right]
$$

\section{MATERIAIS E MÉTODOS}

\section{A. Experimento e coletas}

O experimento foi realizado por homem de 37 anos, saudável, $1,70 \mathrm{~m}$ e $96 \mathrm{~kg}$, utilizando tênis onde o protótipo foi amarrado rigidamente a face ventral do pé pelos cadarços Fig. 2.

Para cada configuração do protótipo a ser estudada, foram realizadas 30 caminhadas em ambiente outdoor (local aberto), no sentido horário, em um circuito retangular fechado de percurso útil total $22,1 \mathrm{~m}$, delimitado por duas linhas laterais distantes aproximadamente $0,4 \mathrm{~m}$ uma da outra Fig. 3. O início das caminhadas se dava no vértice superior esquerdo da figura, sempre com os pés alinhados e imóveis, e terminava com o retorno ao ponto de origem. A cobaia manteve os passos dentro dos limites das faixas, sem que isso afetasse

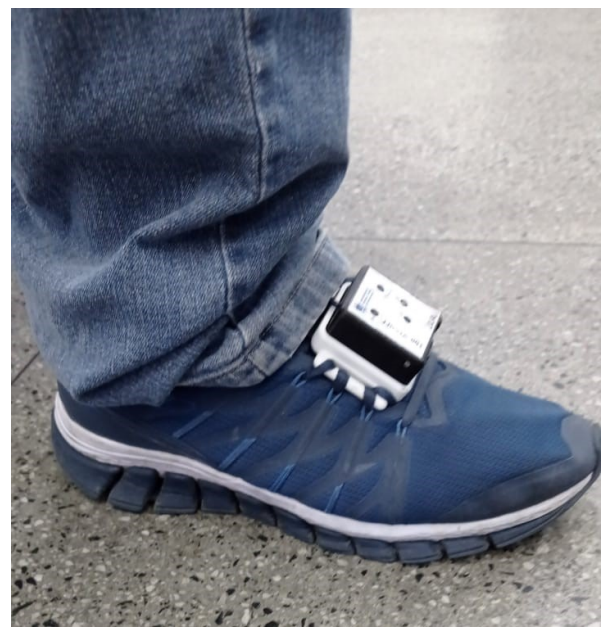

Fig. 2. Protótipo amarrado ao pé pelos cadarços do sapato.

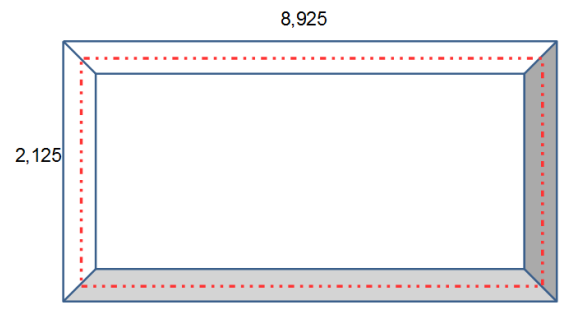

Fig. 3. Esquema do percurso fechado utilizado para coleta de dados. A linha tracejada indica o percurso contabilizado como a distância total percorrida.

o movimento natural da caminhada. No início de cada coleta os pés ficavam aproximadamente 2 segundos imóveis antes de iniciar a caminhada, para inicialização do sistema de navegação inercial.

\section{B. Protótipo do dispositivo}

O protótipo foi montado em uma caixa plástica compacta contendo a placa microcontrolada, as IMUs e uma bateria. A caixa pode ser facilmente acoplada ou desacoplada magneticamente a base amarrada ao tênis.

A placa microcontrolada utilizada no protótipo foi a ESP32. Um dos motivos dessas escolha foi a disponibilidade do Bluetooth integrado que permite a fácil transmissão dos dados coletados a qualquer outro dispositivo Bluetooth pareado próximo, como um telefone celular ou computador, facilitando as coletas e criação dos datasets. A ESP-32 também possui interfaces de comunicação $\mathrm{I}^{2} \mathrm{C}$ de fácil configuração e que foram utilizadas para comunicação com a IMU.

A IMU utilizada foi a MPU-6050 da InvenSense, que possui 3 acelerômetros e 3 giroscópios triaxiais, escolhida pelo seu baixo custo e facilidade de compra. Os fundos de escala do acelerômetro pode ser ajustado para $\pm 2 g, \pm 4 g$, $\pm 8 \mathrm{~g}$ e $\pm 16 \mathrm{~g}$ e dos giroscópios para $\pm 250^{\circ} / \mathrm{s}, \pm 500^{\circ} / \mathrm{s}$, $\pm 1000^{\circ} / \mathrm{s}$ e $\pm 2000^{\circ} / \mathrm{s}$. É importante notar que a MPU-6050 não possui magnetômetros, assim o dispositivo não sofre com 
interferências de massas metálicas ou magnéticas a que for submetido, seja outdoor ou indoor (local fechado).

O protótipo coleta os dados brutos a $100 \mathrm{~Hz}$ e os transmite via Bluetooth para serem salvos. Esses dados são posteriormente processados pelos algoritmos, escritos em Python.

TABELA I

ALGORITMOS

\begin{tabular}{|c|c|c|c|}
\hline Algoritmos & Estados & Correção & Orientação \\
\hline 9IEZ & 9 & ZUPT & Equação (5) \\
9IEZ+MAD & 9 & ZUPT & Equação (26) \\
15IEZ+ZARU & 15 & ZUPT+ZARU & Equação (5) \\
15IEZ+ZARU+MAD & 15 & ZUPT+ZARU & Equação (26) \\
\hline
\end{tabular}

TABELA II COMBINAÇÕES DAS CONFIGURAÇÕES DA IMU

\begin{tabular}{|c|c|c|}
\hline Configuração & Acelerômetro & Giroscópio \\
\hline C00 & $\pm 2 g$ & $\pm 250^{\circ} / s$ \\
C03 & $\pm 2 g$ & $\pm 2000^{\circ} / s$ \\
C30 & $\pm 16 g$ & $\pm 250^{\circ} / s$ \\
C33 & $\pm 16 g$ & $\pm 2000^{\circ} / s$ \\
\hline
\end{tabular}

\section{Algoritmos e combinações}

Para reconstrução da trajetória foram utilizados 4 algoritmos baseados em um sistema de navegação inercial (INS) que utiliza um filtro de Kalman estendido (EKF) para estimar os estados do sistema e corrigi-los em uma atualização de zero velocidade (ZUPT), apresentado na Fig. 1 como IEZ. As variações do algoritmos se dá na quantidade de estados estimados ( 9 ou 15, apresentados em (9) e (18)), nos métodos de correção (ZUPT e ZARU) e de estimativa de orientação. A estimativa de orientação tradicional é dada pela integração das velocidades angulares, dada por (5), e nos algoritmos que utilizam o filtro de Madgwick (abreviado para MAD) a estimativa é calculada por (26). A Tabela I detalha esses pontos.

As configurações definidas para o estudo foram os menores e maiores fundos de escala dos sensores, sendo $\pm 2 g$ e $\pm 16 \mathrm{~g}$ para os acelerômetros e $\pm 250^{\circ} / \mathrm{s}$ e $\pm 2000^{\circ} / \mathrm{s}$ para os giroscópios, combinadas conforme a Tabela II.

Os quatro algoritmos e as quatro configurações resultam nas 16 combinações estudadas.

\section{Métricas de avaliação}

Para avaliar o desempenho das configurações das IMUs e dos algoritmos foram analisados o comportamento dos erros de posicionamento (30) e do erro acumulado de distância (31), onde Dif representa a distância euclidiana entre a posição inicial e final, $D_{T}$ é a distância total percorrida e $D_{E}$ é a distância total estimada. $\mathrm{O}$ erro de posicionamento foi utilizado em [2] e [4] e o erro de distância acumulada em [15] e [16]. Cada medição foi então processada pelos 4 algoritmos para ter trajetórias e erros estimados analisados.

$$
E P=\frac{D i f}{D_{T}}
$$

$$
E D=\frac{D_{T}-D_{E}}{D_{T}}
$$

\section{RESUlTAdos E Discuss Ão}

Os erros medidos das amostras foram analisados com relação a normalidade utilizando o teste de Shapiro-Wilk e os gráficos quantil-quantil. Devido a quantidade de medições, 120 , eventuais falhas nas coletas aconteceram e para reduzir distorções nas análises, medidas de erro fora dos limites de 3 desvios padrão foram desconsiderados.

Todas as trajetórias reconstruídas, divididas por configuração, são apresentadas nas Figuras 4, 5, 6 e 7, sendo os algoritmos utilizados em cada figura, no sentido horário, 9IEZ, 9IEZ+MAD, 15IEZ+ZARU+MAD e 15IEZ+ZARU. É possível verificar na Figuras 4 e 6 como o giroscópio configurado em $\pm 250^{\circ} / \mathrm{s}$ em conjunto com a estimativa das orientação pelo filtro de Madgwick afetaram negativamente a reconstrução das trajetórias. Nas Figuras 5 e 7 é possível visualizar trajetórias mais estáveis, mesmo com algumas sendo anômalas.

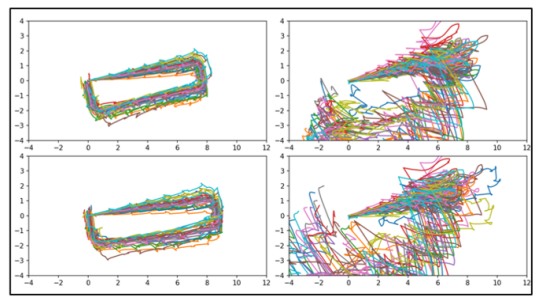

Fig. 4. Reconstrução das trajetórias para configuração C00.

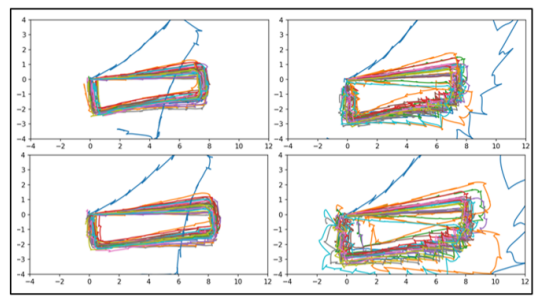

Fig. 5. Reconstrução das trajetórias para configuração C03.

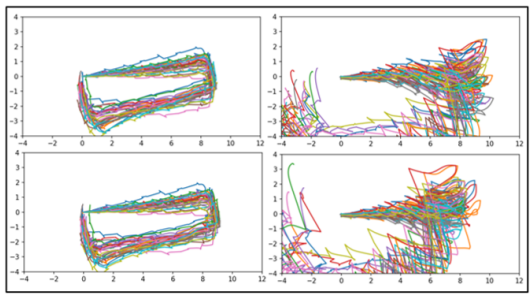

Fig. 6. Reconstrução das trajetórias para configuração C30.

$\mathrm{Na}$ avaliação das combinações entre algoritmos e configurações foram realizados testes $t$ de Welch para verificar a hipótese das médias dos erros serem iguais. Como 


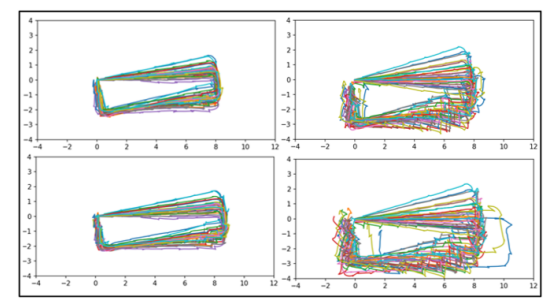

Fig. 7. Reconstrução das trajetórias para configuração C33.

o teste funciona mesmo com amostras de tamanhos diferentes, não foi um problema a exclusão dos pontos discrepantes.

Nas Fig. 8 e Fig. 9 temos os erros estimados por algoritmo utilizando todas as medições realizadas. É possível verificar que os métodos que utilizam o filtro de Madgwick para estimar a orientação possuem intervalos interquartis que apontam variabilidade e mediana elevados em comparação com os outros métodos. Nas Fig. 10 e Fig. 11 são apresentados todos os erros estimados por configurações dos sensores. É possível verificar que a configuração de $\pm 250^{\circ} / \mathrm{s}$ do giroscópio, influencia aumentando as variabilidades e medianas dos erros.

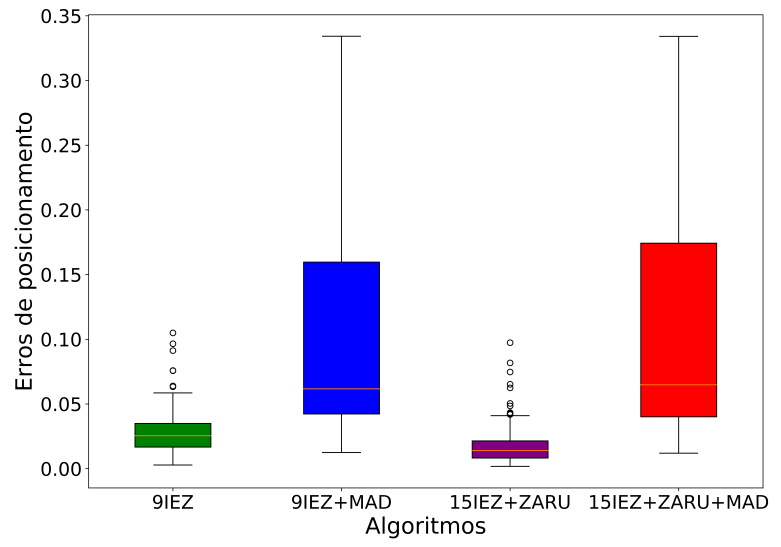

Fig. 8. Diagrama de caixas dos erros de posicionamento por algoritmo.

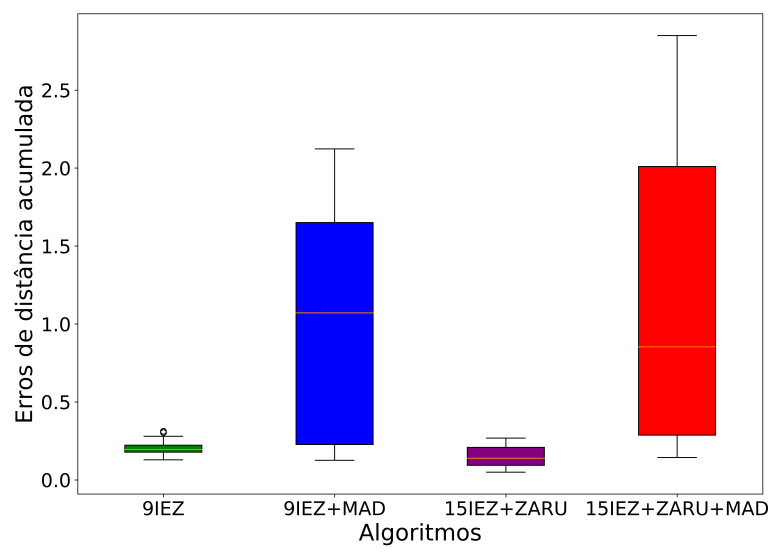

Fig. 9. Diagrama de caixas dos erros de distância acumulada por algoritmo.

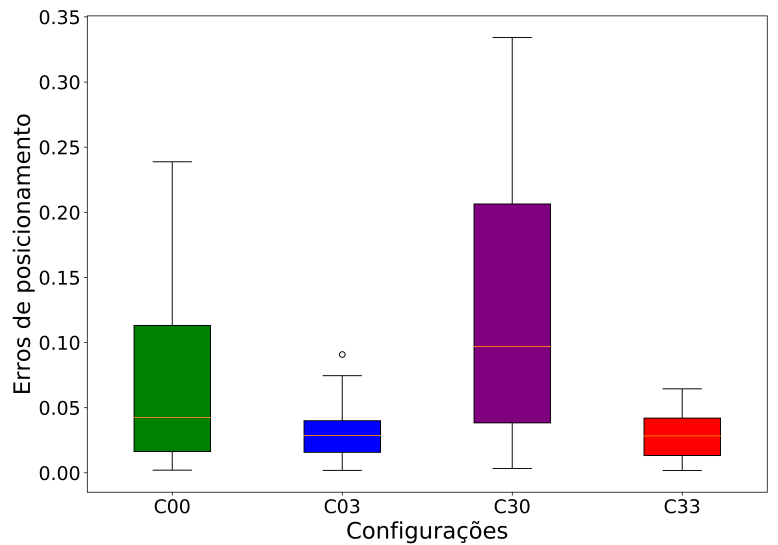

Fig. 10. Diagrama de caixas dos erros de posicionamento por configuração.

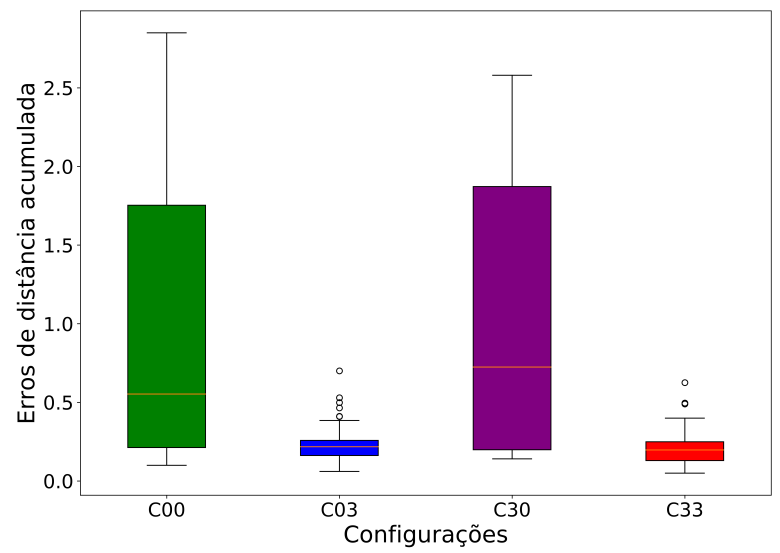

Fig. 11. Diagrama de caixas dos erros de distância acumulada por configuração.

Nas Fig. 12 e Fig. 13 é possível ver uma discrepância nas variâncias e medianas dos intervalos interquartis que combinam o filtro de Madgwick com as configurações dos giroscópios em $\pm 250^{\circ} / \mathrm{s}$. Para os erros de posição as maiores médias ocorreram com os algoritmos 9IEZ+ZARU+MAD e 15IEZ+ZARU+MAD, sendo 0.18287 e 0.22187 para a configuração $\pm 16 g$ e $\pm 2000^{\circ} / \mathrm{s}$, e 0.12573 e 0.12326 para a configuração $\pm 16 \mathrm{~g}$ e $\pm 2000^{\circ} / \mathrm{s}$. Nos mesmos algoritmos ocorreram maiores médias para os erros acumulados de distância, sendo 1.73145 e 2.03735 para a configuração $\pm 16 g$ e $\pm 250^{\circ} / s$, e 1.56811 e 1.94677 para a configuração $\pm 2 g$ e $\pm 250^{\circ} / \mathrm{s}$.

Por outro lado não é tão simples distinguir quais combinações de método e configurações tem melhor desempenho que as outras. Assim foi conduzido um test $t$ de Welch para verificar as médias dos erros e identificar o desempenho dos algoritmos e configurações. As duas menores médias, para ambos os erros estudados, ocorreram com o algoritmo 15IEZ+ZARU e as configurações $\pm 2 g$ e $\pm 2000^{\circ} / s$, e $\pm 16 g$ e $\pm 2000^{\circ} / \mathrm{s}$, e o teste apontou que estatisticamente elas podem ser consideradas iguais. As médias foram de 0.01342 e 0.00982 para os erro de posição e 0.09579 e 0.09566 para o erro de distância acumulada. Todas as médias dos erros de 


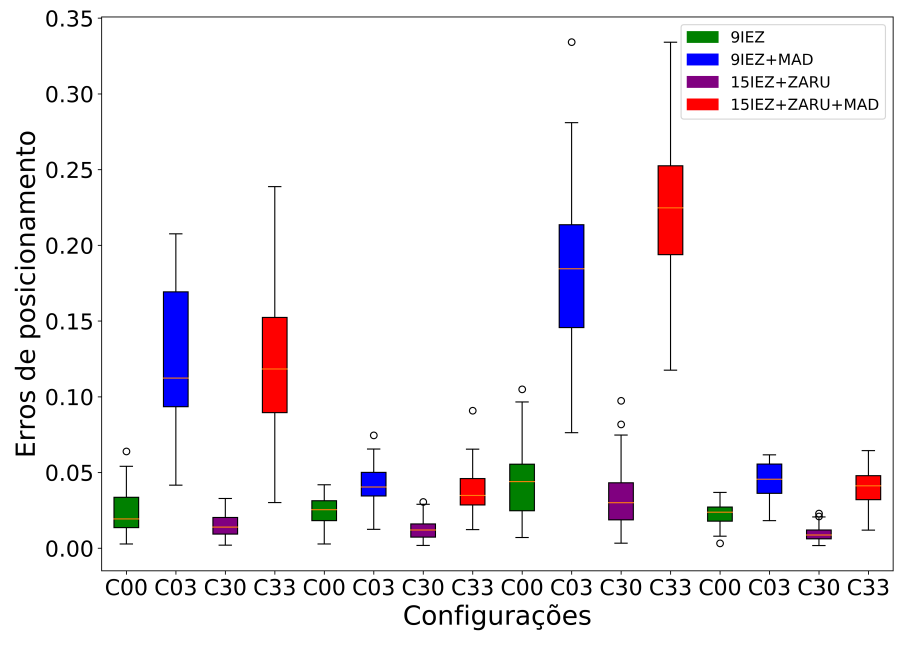

Fig. 12. Diagrama de caixas dos erros de posicionamento por algoritmo e configuração.

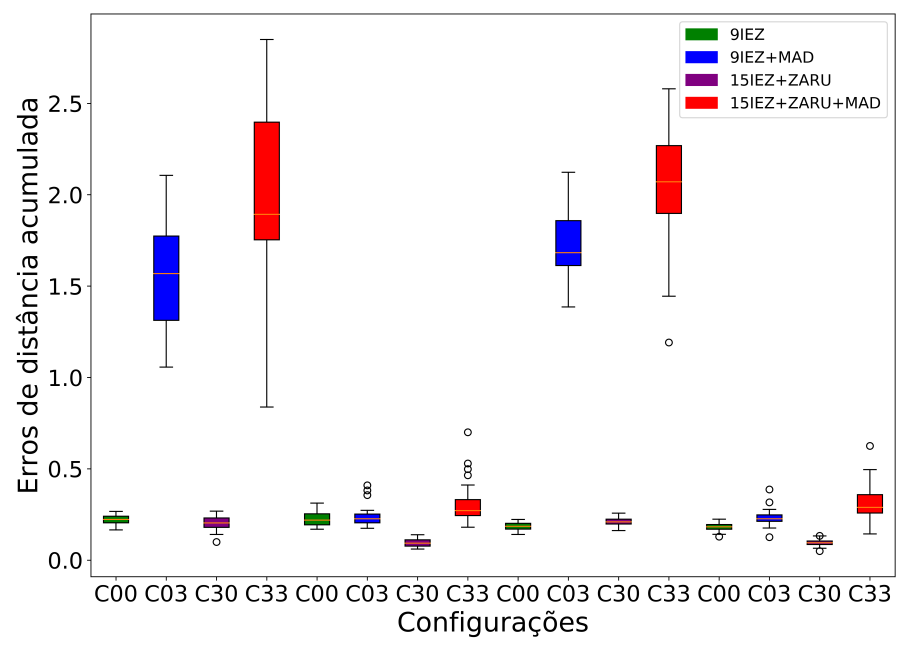

Fig. 13. Diagrama de caixas dos erros acumulados de distância por algoritmo e configuração.

posicionamento podem ser vistas na Tabela III e dos erros de distância acumulada na Tabela IV.

TABELA III

MÉDIA DE ERROS DE POSICIONAMENTO

\begin{tabular}{|c|c|c|c|c|}
\cline { 2 - 5 } \multicolumn{1}{c|}{} & \multicolumn{4}{|c|}{ Configurações } \\
\hline Algoritmos & $\boldsymbol{C 0 0}$ & $\boldsymbol{C 0 3}$ & $\boldsymbol{C 3 0}$ & $\boldsymbol{C 3 3}$ \\
\hline 9IEZ & 0.02477 & 0.02484 & 0.04433 & 0.02263 \\
9IEZ+MAD & 0.12573 & 0.04233 & 0.18287 & 0.04506 \\
15IEZ+ZARU & 0.01471 & 0.01342 & 0.03561 & 0.00982 \\
15IEZ+ZARU+MAD & 0.12326 & 0.03891 & 0.22187 & 0.03966 \\
\hline
\end{tabular}

\section{Conclus Ão E TRABALhos Futuros}

Este trabalho apresentou um estudo sobre as configurações da IMU MPU-6050 e seu desempenho com alguns dos PDRs mais comuns baseados em EKFs. Foram implementados sistemas de navegação que se valeram apenas dos acelerômetros
TABELA IV

MÉdIA DE ERROS DE DISTÂNCIA ACUMULADOS

\begin{tabular}{|c|c|c|c|c|}
\cline { 2 - 5 } \multicolumn{1}{c|}{} & \multicolumn{4}{|c|}{ Configurações } \\
\hline Algoritmos & $\boldsymbol{C 0 0}$ & $\boldsymbol{C 0 3}$ & $\boldsymbol{C 3 0}$ & $\boldsymbol{C 3 3}$ \\
\hline 9IEZ & 0.21927 & 0.22546 & 0.18443 & 0.18053 \\
9IEZ+MAD & 1.56811 & 0.23870 & 1.73145 & 0.23147 \\
15IEZ+ZARU & 0.20237 & 0.09579 & 0.21085 & 0.09566 \\
15IEZ+ZARU+MAD & 1.94677 & 0.31068 & 2.03735 & 0.31387 \\
\hline
\end{tabular}

e giroscópios da IMU, sem auxilio de infraestrutura externa e aplicando as técnicas de correção de erro ZUPT e também ZUPT+ZARU. Essas técnicas, da forma que foram implementadas, só podem ser utilizadas com os sensores montados no pé devido a mecânica da caminhada humana.

Os resultados apontam que na utilização da MPU-6050 deve-se evitar a configuração do giroscópio em $\pm 250^{\circ} / \mathrm{s}$ principalmente se a estimativa de orientação do SNI estiver sendo dada pelo filtro de Madgwick. A configuração com melhores resultados foi a dos maiores fundos de escala, $\pm 16 \mathrm{~g}$ e $\pm 2000^{\circ} / s$, aliada ao algoritmo 15IEZ+ZARU, com uma média de erros de posicionamento de $0.98 \%$ da distância total percorrida, e erros de distância acumulada de $9.5 \%$ da distância total percorrida.

Em trabalhos futuros, deverá ser investigado o comportamento dos erros em outros cenários como longas caminhadas, circuitos assimétricos e locais com desnível. A análise dos erros, das estimativas de posição e trajetória em 3 dimensões, com a utilização de sensores como magnetômetros e barômetros, serão também avaliados.

\section{REFERÊNCIAS}

[1] E. Foxlin, "Pedestrian tracking with shoe-mounted inertial sensors," IEEE Computer graphics and applications, v. 25, n. 6, p. 38-46, 2005.

[2] A. R. Jiménez, F. Seco, J. C. Prieto, J.Guevara, "Indoor pedestrian navigation using an INS/EKF framework for yaw drift reduction and a foot-mounted IMU," In: 2010 7th workshop on positioning, navigation and communication. IEEE, 2010. p. 135-143.

[3] S. Rajagopal, "Personal dead reckoning system with shoe mounted inertial sensors," In Master of Science Thesis, Stockholm, Sweeden, 2008, pp. 1-45.

[4] L. Zhang, Y. Liu, J. Sun, ”A Hybrid Framework for Mitigating Heading Drift for a Wearable Pedestrian Navigation System through Adaptive Fusion of Inertial and Magnetic Measurements,' Applied Sciences, v. 11, n. 4, p. 1902, 2021.

[5] H. Li, H. Guo, Y. Qi, L. Deng, M. Yu, "Research on multi-sensor pedestrian dead reckoning method with UKF algorithm," Measurement, v. 169, p. 108524, 2021.

[6] C. Fischer, P. T. Sukumar, M. Hazas, "Tutorial: Implementing a pedestrian tracker using inertial sensors," IEEE pervasive computing, v. 12, n. 2, p. 17-27, 2012.

[7] J. Nilsson, I. Skog, P. Händel, K. V. S. Hari, "Foot-mounted INS for everybody-an open-source embedded implementation,' In: Proceedings of IEEE/ION PLANS 2012. 2012. p. 140-145.

[8] S. Qiu, Z., Wang, H. Zhao, K., Qin, Z. Li, H. Hu, "Inertial/magnetic sensors based pedestrian dead reckoning by means of multi-sensor fusion," Information Fusion, v. 39, p. 108-119, 2018.

[9] Xin L., Yang W., "Evaluation of AHRS algorithms for foot-mounted inertial-based indoor navigation systems," Open Geosciences, v. 11, n. 1, p. 48-63, 2019.

[10] S. Madgwick, A. Harrison, R. Vaidyanathan, "Estimation of IMU and MARG orientation using a gradient descent algorithm,' In: 2011 IEEE international conference on rehabilitation robotics. IEEE, 2011. p. 1-7. 
[11] R. Mahony, T. Hamel, JM. Pflimlin, ”Nonlinear complementary filters on the special orthogonal group," IEEE Transactions on automatic control, v. 53, n. 5, p. 1203-1218, 2008.

[12] M. L. Hoang, A. Pietrosanto, "Yaw/Heading optimization by drift elimination on MEMS gyroscope," Sensors and Actuators A: Physical, v. 325, p. 112691, 2021.

[13] Y. Wu, H. B. Zhu, Q. X. Du, S. M. Tang, "A survey of the research status of pedestrian dead reckoning systems based on inertial sensors," International Journal of Automation and Computing, v. 16, n. 1, p. 6583, 2019.

[14] X. Hou, J. Bergmann, "Pedestrian dead reckoning with wearable sensors: A systematic review," IEEE Sensors Journal, v. 21, n. 1, p. 143-152, 2020.

[15] X. C. Zhou, J. X.Chen, Y. Dong, X. R. Lu, J. W. Cui, B. Y. Zheng, "Pedestrian navigation with foot-mounted inertial sensors in wearable body area networks," In: Signal and Information Processing Association Annual Summit and Conference (APSIPA), 2014 Asia-Pacific. IEEE, 2014. p. 1-7.

[16] M. V.Potter, L. V. Ojeda, N. C. Perkins, S. M. Cain, "Effect of IMU design on IMU-derived stride metrics for running," Sensors, v. 19, n. 11, p. 2601, 2019. 